\title{
Model-Invariant Hybrid LES-RANS Computation of Separated Flow Past Periodic Hills
}

\author{
Stephen Woodruff
}

\begin{abstract}
The requirement that physical quantities not vary with a hybrid LESRANS model's blending parameter imposes conditions on the computation that lead to better results across LES-RANS transitions. This promises to allow placement of those transitions so that LES is performed only where required by the physics, improving computational efficiency. The approach is applied to separated flow past periodic hills, where good predictions of separation-bubble size are seen due to the gradual, controlled, LES-RANS transition and the resulting enhanced near-wall eddy viscosity.
\end{abstract}

\section{Introduction}

Full realization of the potential of hybrid LES-RANS computations requires that LES regions be placed only where necessary to capture the physics the RANS model cannot, and RANS be used everywhere else for computational efficiency. That current hybrid methods lack this flexibility, even in a simple case like plane channel flow, was demonstrated by Nikitin et al. [13] in 2001. They found that placing the LES-RANS transition in the log layer led to what is now commonly referred to as "log-layer mismatch": the LES log layer is shifted upwards and the mean velocity in the central portion of the channel is too large. Many authors have reported similar results. As a consequence, if a RANS model fails in certain parts of a flow (say near the wall-mounted injector of a scramjet or in a smooth-body separation bubble), one's only alternatives are to accept the errors resulting from the LES-RANS transition or to render the errors neglegible by defining unnecessarily large LES regions with transitions far enough away from the region of interest.

The model-invariant hybrid computation [21,22] addresses this problem by establishing a basis for interpreting the results of a hybrid computation in those parts

Stephen Woodruff

NASA Langley Research Center, Hampton, VA 23681, e-mail: stephen.1.woodruff@nasa.gov 


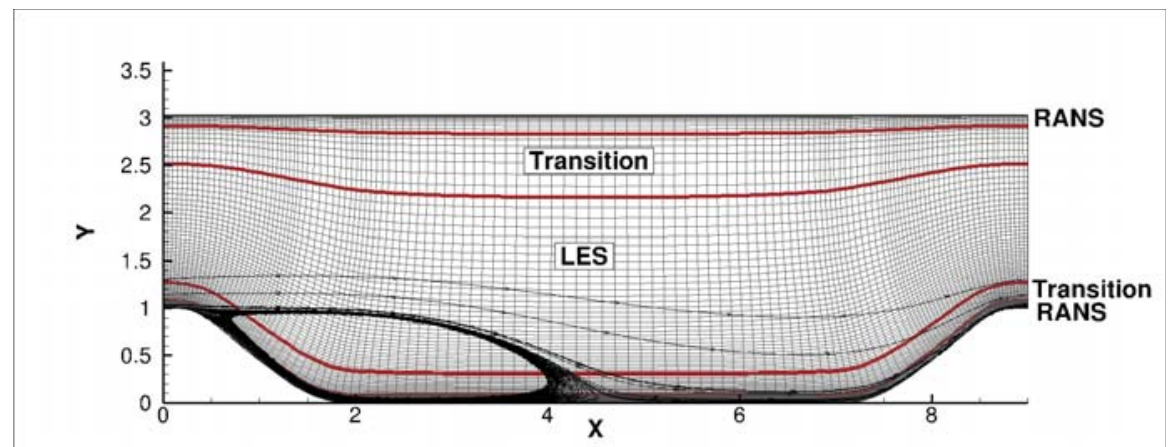

Fig. 1 Flow configuration, showing mesh, edges of RANS-LES transition zones (thick red lines) and streamlines from model-invariant computation.

of the flow where the model is somewhere between a pure RANS model and a pure LES model. A blending parameter characterizes this transition, controlling the mixture of RANS and LES at a given point in the flow. As a non-physical artifact of the turbulence model, the value of the blending parameter should not affect physically meaningful results in the transition region if both RANS and LES are valid there. Model-invariant computations ensure this is the case.

Combinations of flow variables that do not change when the blending parameter is varied are model invariants; physical variables must be expressible in terms of these model invariants. The total turbulent kinetic energy should be a model invariant, for example. Expressing it as the sum of the resolved turbulent kinetic energy and the modeled turbulent kinetic energy yields an approximate model invariant, whose accuracy is limited by the fidelity of the underlying RANS and LES models and by the manner of blending them.

Results of model-invariant computations were given for decaying, isotropic, homogeneous turbulence in Reference [21] and for plane channel flow in Reference [22]. The channel-flow results are particularly compelling: the model-invariant computation has no log-law shift and the mean velocity in the channel center is accurately predicted. Additionally, the model-invariant computation attenuates the modeled shear stress and amplifies the resolved shear stresses across the LES-RANS transition, so the RANS zone is closer to pure RANS and the LES zone is closer to pure LES than for a conventional hybrid computation. Predictions of physical quantities are largely unchanged by varying the height and thickness of the LES-RANS transition or the shape of the blending function.

A few examples of other approaches to facilitating LES-RANS transitions involve stochastic forcing [9], definition of a hybrid filter [6], modifying the RANS eddy viscosity to account for resolved Reynolds stresses $[2,10]$ and modeling commutation error [20]. A discussion of the relation between the present and other approaches may be found in Reference [22].

In the present work, a model-invariant computation is performed for the flow past streamwise-periodic hills in a channel (Fig. 1). The flow separates in the lee 
of each hill and the length of the separation bubble has been particularly difficult to predict accurately with most RANS or hybrid computations. Lengths are nondimensionalized by the hill height and velocities by the bulk velocity over the hill. An artificial body force provides an imposed pressure gradient and is controlled to maintain a constant mass flux through the channel. The flow is incompressible and the Reynolds number $(R e)$, based on bulk velocity and hill height, is 10,595. The thick solid red lines in Fig. 1 represent the edges of the LES-RANS transitions.

LES computations $[3,5,19,23]$ provide a largely consistent reference for comparisons. They yield, for $R e=10,595$, separation at $x_{s} \approx 0.19-0.22$ and reattachment at $x_{r} \approx 4.68-4.72$, though one of the computations of Reference [5] gives $x_{r} \approx 4.56$. Hybrid results $[2,4,7,18]$ are much more dispersed, with most giving separation in the range $0.23-0.6$ and reattachment in the range $4.6-5.8$. The best results include a one-equation hybrid model [2] $\left(x_{r} \approx 4.75\right)$ and one based on an explicit algebraic stress model [7] (several computations with $x_{r} \approx 4.65-4.75$ ). Most hybrid computations, however, like the Spalart-Almaras DES [15] reported in Reference [2] $\left(x_{r} \approx 5.20\right)$, yield separation bubbles that are noticeably too large. RANS computations (as discussed in, for example, References $[1,8,12,14]$ ) generally lead to an overly long separation bubble, with reattachment delayed to as far as $x_{r} \approx 6-8$. Nevertheless, several RANS model improvements $[8,12,14]$ have succeeded in enhancing the level of turbulence in the bulk of the separation bubble and advancing the reattachment point to a more realistic location.

The thin tail of the separation bubble means that large variations in the reattachment location can result from very small changes in the flow field near the wall: existing hybrid techniques in fact predict mean velocities and correlations in the bulk of the flow fairly well. The work of Temmerman et al. [19] shows that wall conditions can significantly affect the reattachment location. It will be seen in what follows that the model-invariant computation intensifies the turbulence in this critical region near the wall ahead of separation and reattachment to reduce the size of the separation bubble.

\section{Model-Invariant Computations}

A model-invariant computation is one in which model invariants are preserved as the computation evolves. It turns out that this is possible only if terms involving derivatives of the hybrid LES-RANS model blending parameter are added to the equations of motion. This is because the flow variables are affected both by flow dynamics and by changes in the blending parameter across the LES-RANS transition; the model-invariant terms are required to cancel the non-physical effects of the changing blending parameter and restore the physical balances of the equations.

These new terms necessarily also involve quantities expressing the sensitivity of the flow variables to changes in the blending parameter. The framework following from the model-invariance concept yields a number of methods for determining these model sensitivities. They may be computed, expensively, by finite differences 
using multiple, simultaneous flow simulations [21]. They may also be determined, more cheaply, from approximations to the model invariants [22]; the only additional computational cost of this approach is the evaluation of the new terms.

The model-invariant computation is set up with gradual transitions between RANS and LES regions, to permit better control. A continuous model [16], valid "in between" RANS and LES, is employed within the transition regions. The continuous model has a blending parameter $\lambda$ that is 0 when the model is in RANS mode and 1 when the model is in LES mode. The RANS and LES models are arbitrary, as is the manner of blending them together. The decomposition of the flow variables into resolved and modelled components is defined by the blended model; no explicit decomposition, such as a filter, is used.

If useful flow information is to be derived from the computation in the transition regions, the computed variables must be connected with physical quantities. Model invariant quantities provide the means for doing so, but model invariance is lost as the equations evolve when the blending parameter varies in space and time. A simple thought experiment shows why: a hybrid computation of a homogeneous flow would show variations of the mean flow variables as the transition from RANS to LES is traversed; the modeled kinetic energy would decrease and the resolved stresses would increase, for example. These variations lead to unphysical gradients in the equations of motion and erroneous results.

To see how to incorporate the variable blending parameter into a hybrid computation without also introducing unphysical gradients, let the blending parameter vary in space and time and connect each point in the computation with an alternative computation conducted with a constant blending parameter of the same value. This second computation has no unphysical gradients, of course, because the blending parameter does not vary. The two computations are connected by the coordinate transformation $t=t^{\prime}, \mathbf{x}=\mathbf{x}^{\prime}, s=\xi-\lambda\left(t^{\prime}, \mathbf{x}^{\prime}\right)$, where $(t, \mathbf{x}, s)$ are the coordinates of the variable- $\lambda$ case and $\left(t^{\prime}, \mathbf{x}^{\prime}, \xi\right)$ are the coordinates of the constant- $\lambda$ case.

Connecting the variable- $\lambda$ and constant- $\lambda$ computations through the coordinate transformation guarantees that properties of the computations will be connected similarly. In particular, the property of model invariance is unaffected by variations in $\lambda$ provided the governing equations conform to the coordinate transformation. This means that time and space derivatives transform according to

$$
\frac{\partial}{\partial t^{\prime}}=\tilde{\partial}_{t} \equiv \frac{\partial}{\partial t}-\frac{\partial \lambda}{\partial t} \frac{\partial}{\partial s} \quad \text { and } \quad \nabla^{\prime}=\tilde{\nabla} \equiv \nabla-(\nabla \lambda) \frac{\partial}{\partial s}
$$

( $\nabla^{\prime}$ is the gradient operator on the primed variables); the new terms in these expressions cancel out the unphysical gradients caused by the variation of $\lambda$.

Employing (1), the continuity and momentum equations become

$$
\tilde{\nabla} \cdot \mathbf{v}=0, \quad \tilde{\partial}_{t} \mathbf{v}+(\mathbf{v} \cdot \tilde{\nabla}) \mathbf{v}=-\tilde{\nabla} p+\tilde{\nabla} \cdot\left[\left(\frac{1}{R e}+v_{t}\right) \tilde{\nabla} \mathbf{v}\right],
$$

where $\mathbf{v}$ is the resolved-scale velocity, $p$ is the resolved-scale pressure and $v_{t}$ is the eddy viscosity. 
The basis for the hybrid model employed in this paper is Strelets' Detached-Eddy Simulation (DES) hybrid model [17], which is in turn based on Menter's SST model [11],

$$
\begin{aligned}
\tilde{\partial}_{t} k+(\mathbf{v} \cdot \tilde{\nabla}) k & =P-\left[(1-\lambda) \beta^{*} \omega k+\lambda k^{3 / 2} /\left(C_{D E S} \Delta\right)\right]+\tilde{\nabla} \cdot\left[\left(\frac{1}{R e}+\sigma_{k} v_{t}\right) \tilde{\nabla} k\right] \\
\tilde{\partial}_{t} \omega+(\mathbf{v} \cdot \tilde{\nabla}) \omega & =\frac{\gamma}{v_{t}} P-\beta \omega^{2}+\tilde{\nabla} \cdot\left[\left(\frac{1}{R e}+\sigma_{\omega} v_{t}\right) \tilde{\nabla} \omega\right] .
\end{aligned}
$$

The production $P$ is expressed as $P=v_{t} \tilde{S}_{i j} \tilde{S}_{i j}$, where $\tilde{S}_{i j}$ is the symmetric part of the tensor $\tilde{\nabla} \mathbf{v}$. The Strelets DES expression for the dissipation term in the kinetic energy equation has been replaced by a blended combination of the RANS and LES dissipation terms to make it a blended, continuous, model. The transition to LES occurs below the outer part of the boundary layer where the $k-\varepsilon$ branch would be active, so it is not included. Standard $k-\omega$ constants [11] are employed.

The derivatives of the flow variables with respect to $s$, the model sensitivities appearing in (1), are determined by means of the crude approximation employed previously for plane channel-flow turbulence [22]. The destruction terms in the $k$ and $\omega$ equations are both considered to be model invariants, because they are sinks for the total amounts (resolved and modelled) of these quantities. Differentiating these terms with respect to $s$ and setting the results to zero yields expressions for $k_{s}$ and $\omega_{s}$. If the total kinetic energy $k+|\mathbf{v}|^{2} / 2$ (modeled plus resolved kinetic energies) is also a model invariant, differentiating it yields $|\mathbf{v}|_{s}$ in terms of $k_{s}$. It remains to split $|\mathbf{v}|_{s}$ into components: this is properly done through model invariants based on components of the Reynolds stress tensor, but in this crude approximation, the total $|\mathbf{v}|_{s}$ is simply split into components according to the empirically determined proportions 30\% spanwise, $70 \%$ normal and $0 \%$ streamwise.

Computations are performed with a modification of the code employed in References [21, 22]. Streamwise and normal directions are discretized by fourth-order finite differences and the spanwise direction is discretized spectrally. Second-order time advancement is performed via Newton iteration. Continuity is imposed at each time step; this also requires iteration, due to the presence of the model-invariant terms. The grid of Fröhlich et al. [5] was adapted to yield a $128 \times 84$ streamwisenormal two-dimensional grid that retains the original near-wall normal spacing. Twenty spectral modes are employed in the spanwise direction. A Spalart-Almaras DES [15] computation was performed for validation and yielded results similar to those found previously [2], with reattachment at $x_{r} \approx 5.2$.

\section{Results}

The results of the model-invariant computation just outlined are now compared with a non-model-invariant computation which is the same in all respects except that the model-invariant terms are not included. All quantities presented are averaged over 
the spanwise direction and over a period of 50 flow-through times (based on bulk velocity and channel length), beginning after time averages of the velocity field have settled to fixed values.

Plots of the streamwise velocity contours for the non-model-invariant (Fig. 2) and the model-invariant (Fig. 3) computations show the flow separating at approximately the same point $\left(x_{r} \approx 0.24\right.$ and $x_{r} \approx 0.23$, respectively) but reattaching at $x_{r} \approx 4.78$ in the non-model-invariant computation and $x_{r} \approx 4.67$ in the model-invariant computation. As noted in the Introduction, the bulk of existing hybrid techniques do noticeably worse.

Both the non-model-invariant (Fig. 4) and model-invariant (Fig. 5) modelled kinetic-energy contour plots show spots of high kinetic energy at the inception of the free shear layer defining the separation bubble; the spot is more localized to the transition and RANS layers in the model-invariant computation. The modelinvariant computation also shows increased kinetic energy in the RANS layer in regions ahead of the separation and reattachment points. (It should be noted that the peak kinetic energy, at the inception of the free shear layer, is approximately 0.02 in both cases; the contour levels have been chosen to bring out differences in the two plots elsewhere in the flow.)

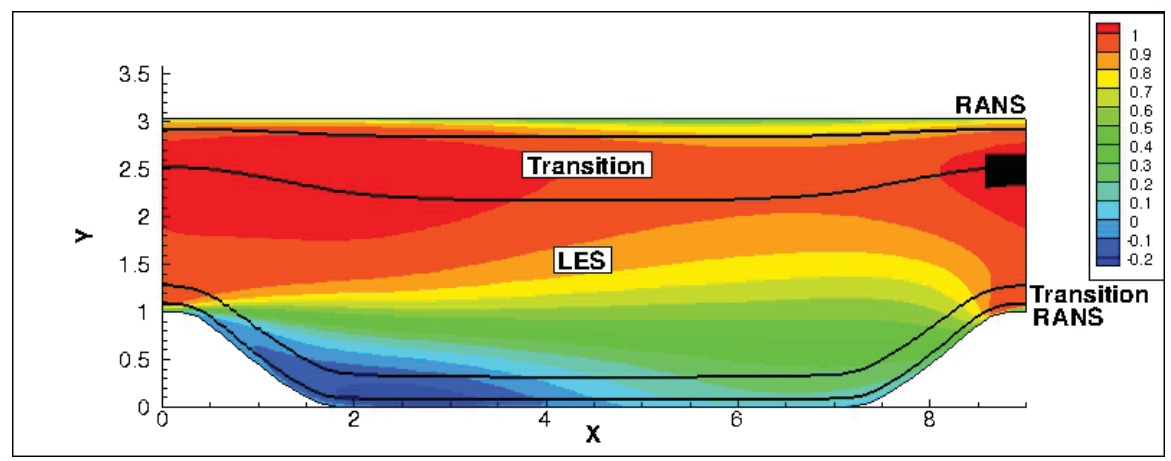

Fig. 2 Streamwise velocity contours for non-model-invariant hybrid computation.

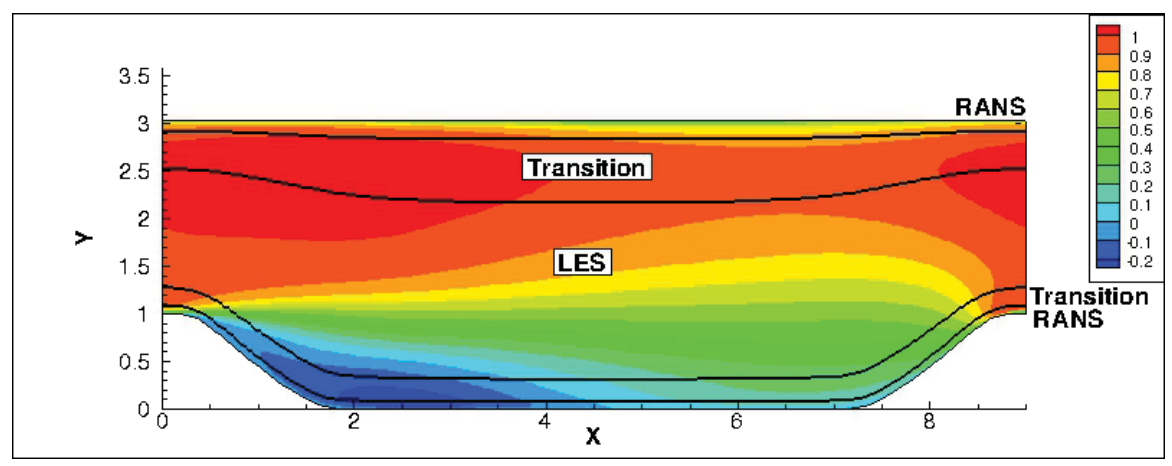

Fig. 3 Streamwise velocity contours for model-invariant hybrid computation. 
Profiles of flow quantities further indicate that the model-invariant terms have a minimal effect in the LES region, but increase the RANS eddy viscosity upstream of the separation and reattachment points. First, cross-channel profiles of the mean streamwise velocity, mean normal velocity, streamwise normal Reynolds stresses and the Reynolds shear stress are given at $x=0.05$, just upstream of the separation point (Fig. 6). The computations with and without the model-invariant terms yield very similar results, except for minor differences in the RANS region near the wall. Both computations agree fairly well with the LES of Fröhlich et al. [5], though the magnitude of the second-order correlations are somewhat low. Profile comparisons are similar at other streamwise locations.

Profiles of the mean contribution of the model-invariant terms to the streamwise momentum, normal momentum, $k$ and $\omega$ equations at $x=0.05$ are shown in Fig. 7 . The mean eddy viscosity is also shown to illustrate how the model-invariant contributions to the $k$ and $\omega$ equations affect separation and reattachment. For the most part, the model-invariant terms act as one would expect to speed the transition from RANS to LES: the terms serve to increase the resolved velocity and decrease the modelled kinetic energy. They also act to reduce the rate of decrease of $\omega$ and the modelled kinetic energy is increased at the RANS edge of the transition region. The

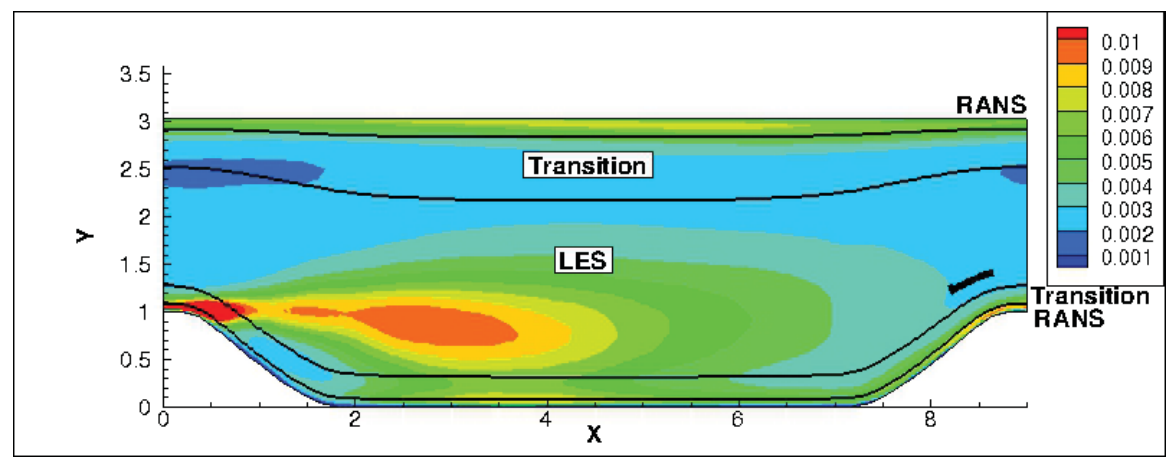

Fig. 4 Modelled kinetic-energy contours for non-model-invariant hybrid computation.

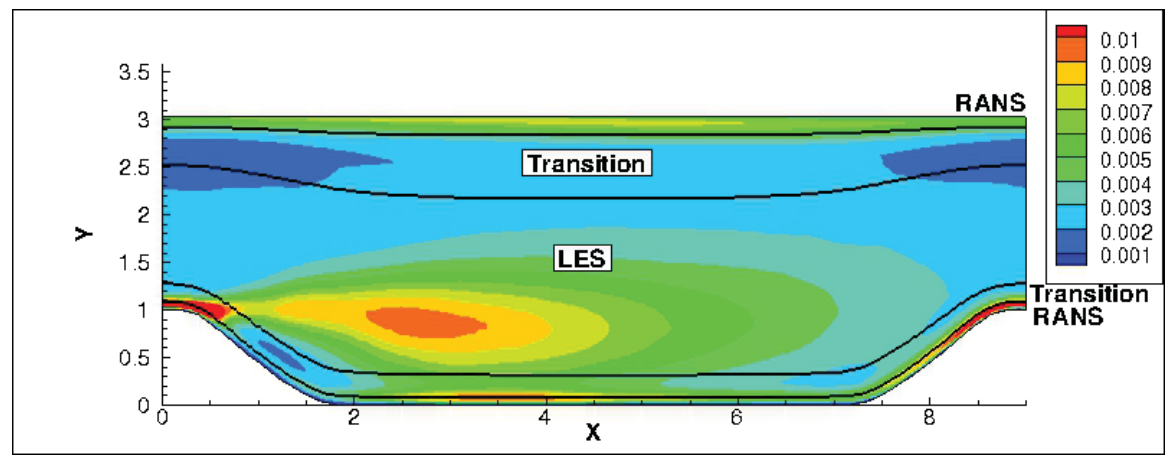

Fig. 5 Modelled kinetic-energy contours for model-invariant hybrid computation. 

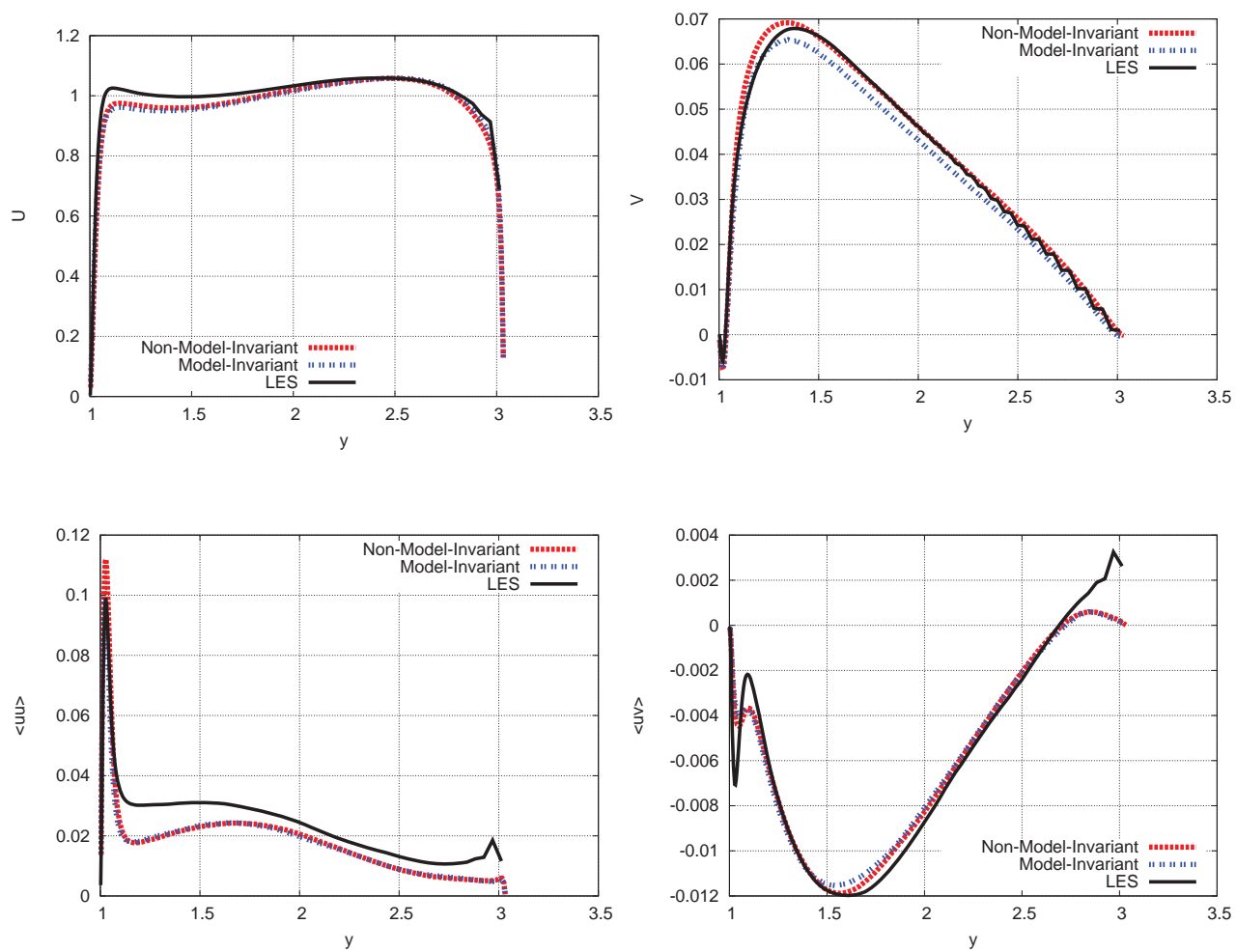

Fig. 6 Moment profiles at $x=0.05$ of non-model-invariant and model-invariant computations compared with LES [5].

mean eddy viscosity profile shows that the latter actions help to localize the eddyviscosity to the RANS layers in the model-invariant computation and to significantly increase, by $50 \%$, the peak eddy viscosity in the model-invariant computation at this location just upstream of the separation point. The increased modelled kinetic energy upstream of the reattachment point (Fig. 5) indicates a similar enhancement of the eddy viscosity occurs there.

In general, higher turbulence levels delay separation and advance reattachment. For Boussinesq-approximation RANS models like that used here, higher turbulence levels correspond to a higher eddy viscosity, and enhancing the eddy viscosity was the mechanism for improving RANS separated-flow predictions in References $[12,14]$. The present model-invariant computation succeeds by enhancing the eddy viscosity in the RANS layer upstream of the reattachment point, moving it forward. It also does so upstream of the separation point, moving it slightly downstream. Unlike the channel-flow computations of Reference [22], the turbulence levels in the LES region of the non-model-invariant computation were fairly close to those of the pure LES and are largely unaffected by the addition of the model-invariant terms. 

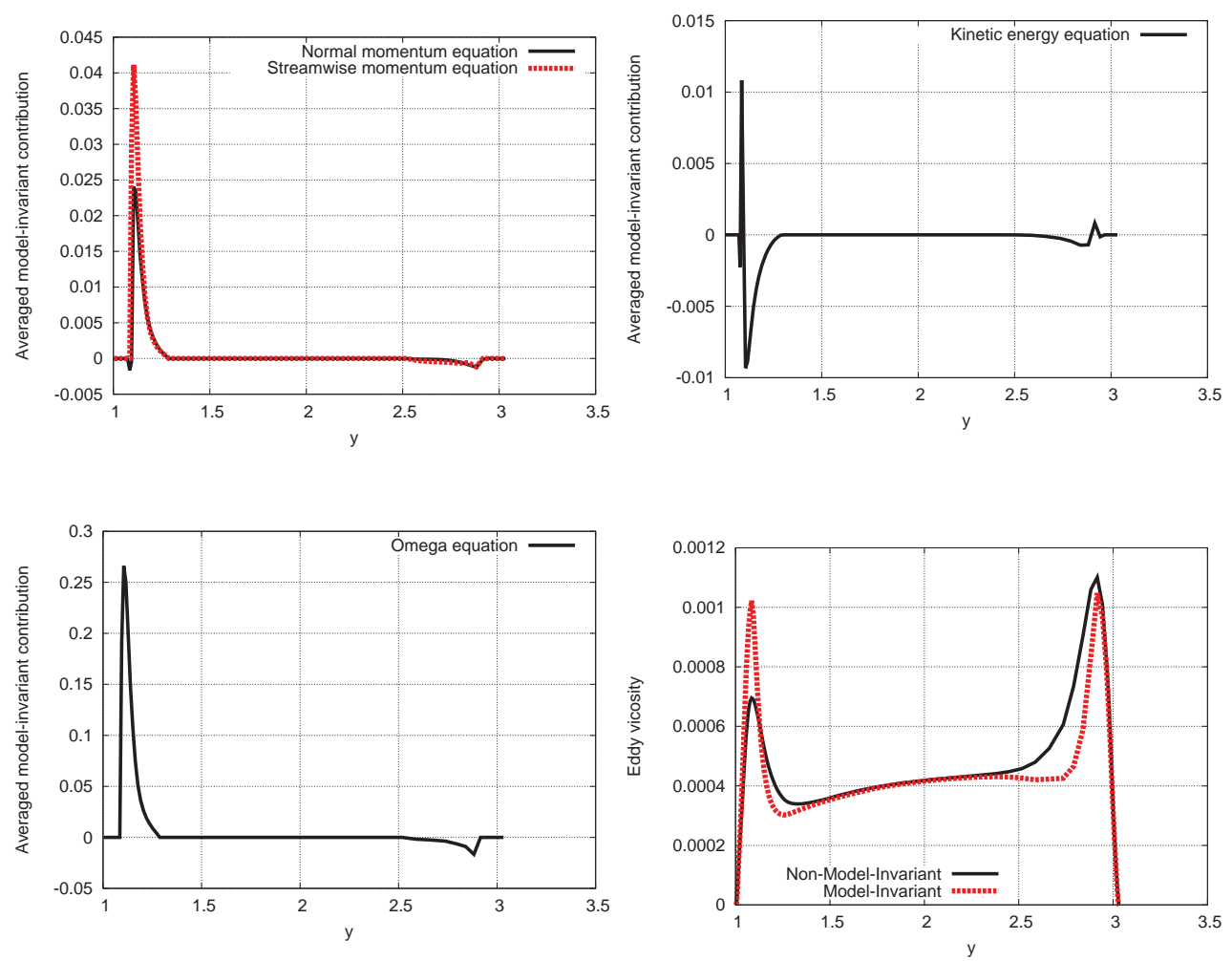

Fig. 7 Mean contribution of model-invariant terms to streamwise and normal momentum equations, kinetic-energy equation and $\omega$ equation and mean eddy viscosity at $x=0.05$

\section{Conclusion}

The observation that physically meaningful quantities cannot depend on the blending parameter in a hybrid LES-RANS computation led to the need to accommodate variations in the blending parameter in the derivatives of the governing equations. The concept of model invariance provides a framework for understanding this and other aspects of hybrid computations, as well as a means for computing the model sensitivities that arise in the new terms.

The model-invariant computation of flow past periodic hills led to significant improvements in flow predictions compared to SA DES and many other hybrid computations in the literature. The success of the non-model-invariant computation indicated a significant part of the improvement is due to the gradual LES-RANS transition; the model-invariant terms further reduced the size of the separation bubble. Examination of resolved and modeled turbulence quantities indicated how the introduction of the model-invariant terms led to enhanced eddy viscosity in the RANS layer upstream of the separation and reattachment points, delaying and advancing them, respectively. 


\section{References}

1. Abe, K., Jang, Y.-J., and Leschziner, M. A. (2003) An investigation of wall-anisotropy expressions and length-scale equations for non-linear eddy-viscosity models. Heat and Fluid Flow 24:181-198

2. Breuer, M., Jaffrezic, B., and Arora, K. (2008) Hybrid LES-RANS technique based on a oneequation near-wall model. Theoretical and Compuatational Fluid Dynamics. 22:157-187

3. Breuer, M., Peller, N., Rapp, C., and Manhart, M. (2009) Flow over periodic hills - Numerical and experimental study in a wide range of Reynolds numbers. Computers \& Fluids 38:433-457

4. Fröhlich, J., and von Terzi, D. (2008) Hybrid LES/RANS methods for the simulation of turbulent flows. Progress in Aerospace Sciences 44:349-377

5. Fröhlich, J., Mellen, C. P., Rodi, W., Temmerman, L., and Leschziner, M. A. (2005) Highly resolved large-eddy simulation of separated flow in a channel with streamwise periodic constrictions. Journal of Fluid Mechanics 526:19-66

6. Germano, M. (2004) Properties of the Hybrid RANS/LES Filter. Theoretical and Computational Fluid Dynamics 17:225-231

7. Jaffrezic, B. and Breuer, M. (2008) Application of an Explicit Algebraic Reynolds Stress Model within a Hybrid LES-RANS Method. Flow, Turbulence and Combustion 81:415-448

8. Jakirlic, S. and Maduta, R. (2014) On "Steady" RANS Modeling for improved Prediction of Wall-bounded Separation. AIAA 2014-0586

9. Keating, A., De Prisco, G. and Piomelli, U. (2006) Interface conditions for hybrid RANS/LES calculations. Heat and Fluid Flow 27:777-788

10. Medic, G., Templeton, J. A. and Kalitzin, G. (2006) A Formulation for Near-Wall RANS/LES Coupling. International Journal of Engineering Science 44:1099-1112

11. Menter, F. R. (1994) Two-Equation Eddy-Viscosity Turbulence Models for Engineering Applications. AIAA Journal 32:1598-1605

12. Menter, F. R. and Egorov, Y. (2010) The Scale-Adaptive Simulation Method for Unsteady Turbulent Flow Predictions. Part 1: Theory and Model Description. Flow, Turbulence and Combustion 85:113-138

13. Nikitin, N. V., Nicoud, F., Wasistho, B., Squires, K. D., and Spalart, P. R. (2000) An approach to wall modelling in large-eddy simulations. Physics of Fluids 12:1629-1632

14. Rumsey, C. (2009) Exploring a Method for Improving Turbulent Separated-Flow Predictions with $k$ - $\omega$ Models. NASA TM-2009-215952

15. Spalart, P. R. (2009) Detached-Eddy Simulation. Annual Review of Fluid Mechanics 41:181202

16. Speziale, C. G. (1998) Turbulence Modeling for Time-Dependent RANS and VLES: A Review. AIAA Journal 36:173-184

17. Strelets, M. (2001) Detached Eddy Simulation of Massively Separated Flows. AIAA 20010879

18. Temmerman, L., Hadziabdic, M., Leschziner, M. A., and Hanjalic, K. (2005) A Hybrid TwoLayer URANS-LES Approach for Large Eddy Simulation at High Reynolds Numbers. Heat and Fluid Flow 26:173-190

19. Temmerman, L., Leschziner, M. A., Mellen, C. P., and Fröhlich, J. (2003) Investigation of Wall-Function Approximations and Subgrid-Scale Models in Large-Eddy Simulation of Separated Flow in a Channel with Streamwise Periodic Constrictions. Heat and Fluid Flow 24:157-180

20. Wallin, S. and Girimaji, S. (2009) Commutation Error Mitigation in Variable-Resolution PANS Closure: Proof of Concept in Decaying Isotropic Turbulence. AIAA 2011-3105

21. Woodruff, S. L. (2010) Coupling Turbulence in Hybrid LES-RANS Techniques, Seventh International Symposium on Turbulence and Shear-Flow Phenomena, Ottawa, Canada

22. Woodruff, S. L. (2013) A New Formulation for Hybrid LES-RANS Computations. AIAA 2013-2722

23. Ziefle, J., Stolz, S., and Kleiser, L. (2008) Large-Eddy Simulation of Separated Flow in a Channel with Streamwise-Periodic Constrictions. AIAA Journal 46:1705-1718 